\title{
Superficial Siderosis From Radiation-Induced Cavernoma of Brain
}

\author{
Kenneth Montini ${ }^{a}$, Krishnamoorthy Thamburaj ${ }^{\mathrm{a}, \mathrm{b}}$
}

\begin{abstract}
We report a unique case of superficial siderosis (SS) from radiationinduced cavernous angioma of brain. Cavernous malformations of brain and SS were identified 9 years after the patient underwent resection of fourth ventricle medulloblastoma along with craniospinal radiation and chemotherapy. His most recent clinical assessment at the age of 23 years, revealed bilateral high-frequency sensory neural hearing loss at audiology along with gaze-evoked nystagmus and slight impairment of tandem walking.
\end{abstract}

Keywords: Superficial siderosis; Cavernous angioma; Cavernoma; Radiation

\section{Introduction}

Cavernous malformations of the central nervous system are considered to be developmental vascular malformations, and majority of them are asymptomatic. Symptomatic cavernomas mainly present with seizures, headache or bleeding with associated deficits [1]. Occasionally they may result in superficial siderosis (SS) of central nervous system $[2,3]$. We report a unique case of SS from radiation-induced cavernous angioma.

\section{Case Report}

A 23-year-old male was undergoing regular neurosurgical evaluation at our institute after he underwent resection of medulloblastoma of fourth ventricle. In July 1997 at the age of 9 years, he underwent resection of the tumor through

Manuscript accepted for publication May 28, 2014

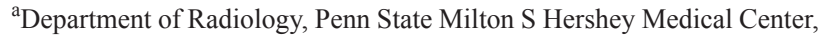
Penn State College of Medicine, Hershey, PA 17033, USA

${ }^{\mathrm{b}}$ Corresponding Author: Krishnamoorthy Thamburaj, Department of

Radiology, Penn State Milton S Hershey Medical Center, Penn State

College of Medicine, Hershey, PA 17033, USA.

Email: kthamburaj@hmc.psu.edu

doi: http://dx.doi.org/10.14740/jnr273w suboccipital craniectomy (Fig. 1A). Prior to surgery, he underwent ventriculoperitoneal shunting. In the post-operative period, he was treated with 23.4 Gy radiotherapy to the craniospinal axis and 54 Gy (total) boost to the posterior fossa over 3 months from July to September 1997. Chemotherapy was administered with eight weekly doses of vincristine. His maintenance therapy consisted of eight cycles of CCNU, cisplatin and vincristine. He received a total of $487.5 \mathrm{mg} / \mathrm{m}^{2}$ of cisplatin. He completed his chemotherapy in September 1998. Since then he was undergoing neurosurgical evaluation and MRI of brain at regular intervals. He developed pituitary dysfunction from craniospinal radiation with growth hormone, cortisol and thyroid deficiency. He had been placed on regular cortisol and thyroid replacement. Until the audiology evaluation in 2005, his neurological examination identified no focal deficits with intact intellectual function. Also, the MRI of brain done at regular intervals demonstrated stable post-operative changes with no evidence of tumor recurrence. No other abnormalities were identified on MRI of brain. An audiology examination done in November 2005 revealed bilateral sensorineural hearing loss. MRI of brain obtained in June 2006 identified a new focus of hemorrhage in the right occipital lobe with no documented neurological deficits. A follow-up MRI of brain obtained 3 months later revealed evolutionary changes of an underlying cavernoma. Multiple cavernomas in the cerebral hemispheres were also seen with many of them concentrated in bilateral occipital and temporal lobes. Subsequent MRI studies of brain demonstrated features of SS from pial hemosiderin staining of the brainstem and the cerebellar folia (Fig. 1B, C). There was no tumor recurrence.

His most recent neurological examination in December 2011 identified gaze-evoked nystagmus. He had bilateral high frequency hearing loss. There was slight impairment of tandem gait with normal finger to nose testing. His strength was $5 / 5$ in all extremities. Facial sensation was intact. He is helping his father in his business without difficulty.

\section{Discussion}

SS is the result of hemosiderin deposition within the sub- 


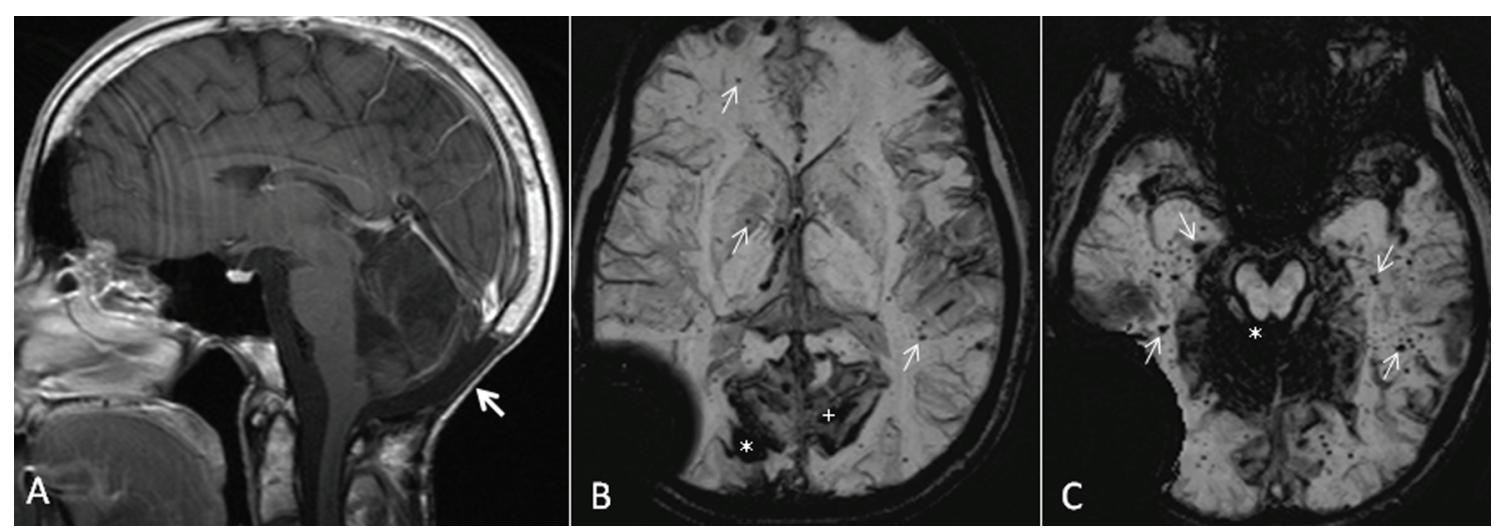

Figure 1. (A) Sagittal T1 post-gadolinium image demonstrates post-operative changes of suboccipital craniectomy and resection of medulloblastoma in the posterior fossa (arrow). (B) Axial susceptibility-weighted minimum intensity pixel (mIP) image demonstrates prominent signal drop from the right occipital cavernoma $\left(^{*}\right)$. Note the prominent susceptibility signals in the adjacent sulci in bilateral medial parieto-occipital regions from intense pial hemosiderin staining (+). Also, note the multiple tiny signal dropouts scattered throughout the brain from small cavernomas and telangiectasia, some of them marked with arrows. The large signal drop in the right temporopariteal region extending outside the calvarium is from the shunt reservoir. (C) Axial susceptibility-weighted $\mathrm{mIP}$ image demonstrates superficial siderosis causing signal drop in the cerebellar folia and the pial surface of brainstem (*). Also note numerous tiny signal dropouts in the bilateral temporal lobes and occipital lobes from radiation-induced cavernomas and telangiectasia (arrows).

pial layers of the central nervous system as a result of recurrent bleeding into the subarachnoid space [4]. In many cases, the cause of SS may remain unknown after extensive investigations $[4,5]$. Among the well-known causes, SS is most commonly caused by a spinal dural defect in $47 \%$ of cases. Tumors including ependymoma, oligodendroglioma, pineocytoma and paraganglioma are responsible for $35 \%$ of SS. Vascular malformations including cavernous angioma, arteriovenous malformation and aneurysm are responsible for $18 \%$ of cases of SS [4].

Cavernous malformations are also called as cavernous angiomas, cavernomas or cryptic vascular malformations [1]. They are well known to develop after cranial irradiation $[6,7]$. Baumgartner et al reported development of cavernous angiomas 7 - 19 years after cranial irradiation [6]. In a literature review of radiation-induced cavernomas, Nimjee et al found reports in the literature of 76 patients with radiation-induced cavernomas with a mean latency of 8.9 years following treatment [7]. Histologically, cavernomas demonstrate compact mass of sinusoids lined by single layer of endothelial cells with intervening collagenous or fibrous stroma [2]. The radiation-induced cavernous angiomas are histologically indistinguishable from sporadic lesions and they can grow to become symptomatic within 5 years [6]. In our case, review of the medical records dating back to June 1997 did not establish the presence of cavernous angiomas in spite of several MRI studies dating from June 1997. Presence of cavernomas was established in our case on MRI 8 years after receiving craniospinal irradiation for the treatment of medulloblastoma. In our case among the multiple cavernomas, occurrence of hemorrhage in the right occipital lobe and the intense pial staining of hemosiderin near the le- sion, establish its role in the development of SS.

Recurrent subclinical bleeding into the subarachnoid space is essential in the development of SS [4]. Hemosiderin deposition is seen in the subpial, subependymal and leptomeningeal layers as coarse deposits in macrophages [4]. Hemosiderin deposition leads to the development of discoloration of leptomeninges and $3 \mathrm{~mm}$ depth of brain, identified in pathological specimens. The dura is spared from hemosiderin deposition. Discoloration or pigmentation is typically seen in cerebellar vermis, folia, basal frontal, brainstem and cranial nerve nuclei. From animal experiments, Koeppen et al proposed a fundamental role for Bergmann glia and ferritin-containing microglia in the cerebellar molecular layer in the development of SS [8]. Neurological manifestations of SS reflect the preferential deposition of hemosiderin. Bilateral sensory neural hearing loss is the characteristic clinical presentation of SS. Other common features include cerebellar ataxia and pyramidal signs. Deposition of hemosiderin on the surface of spinal cord may lead to development of myelopathy in some cases with sensorimotor deficits and bladder involvement. Approximately $27 \%$ of patients may become bed-bound after 11 years [4].

MRI is the best available imaging technique to diagnose SS [5]. Pial and ependymal hemosiderin staining typically gives rise to hypointense layer of signals best demonstrated with gradient echo sequence. If one pays adequate attention to the cranial nerves II, V, VII and VIII, the hypointense signals from these nerves can be recognized easily. Diagnosis can be easily missed if one fails to use the appropriate sequence. Recently available susceptibility-weighted sequence should further improve the diagnosis of this condition. Advanced cases of SS may result in atrophic changes in the cer- 
ebellum. Neuroimaging evaluation of SS does not stop with the identification of SS. It is essential to assess the entire neuraxis including the spine to evaluate for dural leaks and myxopapillary ependymoma [7].

Our unique case consists of the development of SS secondary to a cavernoma, which developed after radiation therapy for medulloblastoma. To the best of our knowledge, the development of SS secondary to radiation-induced cavernoma is a highly unusual presentation.

\section{Conflict of Interest}

The authors report no conflict of interest.

\section{References}

1. Kondziolka D, Lunsford LD, Kestle JR. The natural history of cerebral cavernous malformations. J Neurosurg. 1995;83(5):820-824.

2. Hsu WC, Loevner LA, Forman MS, Thaler ER. Superficial siderosis of the CNS associated with multiple cavernous malformations. AJNR Am J Neuroradiol. 1999;20(7):1245-1248.

3. Kumar A, Aggarwal S, Willinsky R, TerBrugge KG. Posterior fossa surgery: an unusual cause of superficial siderosis. Neurosurgery. 1993;32(3):455-457; discussion 457.

4. Fearnley JM, Stevens JM, Rudge P. Superficial siderosis of the central nervous system. Brain. 1995;118 ( Pt 4):1051-1066.

5. Kumar N. Neuroimaging in superficial siderosis: an indepth look. AJNR Am J Neuroradiol. 2010;31(1):5-14.

6. Baumgartner JE, Ater JL, Ha CS, Kuttesch JF, Leeds NE, Fuller GN, Wilson RJ. Pathologically proven cavernous angiomas of the brain following radiation therapy for pediatric brain tumors. Pediatr Neurosurg. 2003;39(4):201-207.

7. Nimjee SM, Powers CJ, Bulsara KR. Review of the literature on de novo formation of cavernous malformations of the central nervous system after radiation therapy. Neurosurg Focus. 2006;21(1):e4.

8. Koeppen AH, Dickson AC, Chu RC, Thach RE. The pathogenesis of superficial siderosis of the central nervous system. Ann Neurol. 1993;34(5):646-653. 